\title{
NOTE ON DEGREES OF PARTIAL FUNCTIONS ${ }^{1}$
}

\author{
JOHN MYHILL
}

The notion of one function's being recursive in another is normally considered only for full functions; but Davis $[1$, p. 171] has given a definition applicable also to partial functions. For one-argument functions (to which we restrict ourselves for the sake of simplicity) this reads: $f$ is partial recursive in $g$ if there is a completely computable functional ${ }^{2} \Phi$ for which $f(x)=\Phi(g, x)$. And here $\Phi$ is called completely computable if for some partial recursive $h$ we have

$$
\Phi(g, x)=t \leftrightarrow(\exists y)\left(y^{[1]} \subseteq g \text { and } h(x, y)=t\right),
$$

$\left\{i^{[1]}\right\}$ being an effective enumeration of finite functions. In this note we shall argue that Davis' definition does not do justice to our intuitive idea of relative computability; we shall suggest an alternative definition; and we shall show that on either definition there are degrees which are not degrees of any full function. ${ }^{3}$ In fact we shall show that in a suitable sense "almost no" degrees are degrees of full functions. ${ }^{4}$

Let $\gamma$ be any nonrecursive set, and let $g_{0}(2 x)=0$ for $x \in \gamma, g_{0}(2 x+1)$ $=0$ for $x \notin \gamma, g_{0}(x)$ undefined otherwise. Let $c_{\gamma}$ be the characteristic function of $\gamma$. Then $c_{\gamma}(x)$ is 0 if $g_{0}(2 x)$ is 0 and 1 if $g_{0}(2 x+1)$ is 0 , so clearly $c$ is effectively computable from $g_{0}$ in the intuitive sense. None the less, since $g_{0}$ is a restriction of the constant function $n(x)=0$,

Received by the editors August 1, 1960.

1 This research was supported by NSF grant G-7277.

${ }^{2}$ We use the notation of [1] except that (1) upper-case Greek letters denote functionals; (2) lower-case Greek letters denote sets (of numbers); (3) upper-case italic letters denote classes (of sets) as well as relations; (4) the complement of a set $\alpha$ is written $\alpha^{\prime}$; (5) sometimes we write $[\Phi f]$ for $\lambda x \Phi(f, x)$.

3 This fact was known to Lacombe and Shoenfield as early as 1958; but the use of a category argument to establish it, and the consequent strengthening of the result, is new.

4 We say that almost no semicharacteristic functions (see below) have a certain property, if the class of all sets whose semicharacteristic functions have this property is of first category; and that almost no (weak) degrees have a certain property if almost no semicharacteristic functions belong to (weak) degrees having that property. The latter usage is justified by the fact that every weak degree $\mathfrak{D}$ contains a semicharacteristic function (for example $c_{\alpha}^{0}$ where $\alpha=\left\{2^{x} \cdot 3^{f(x)} \mid f(x)\right.$ is defined $\}$ for any $f \in \mathfrak{D}$. (Not every strong degree contains a semicharacteristic function; for example this is not true, by the argument of the following paragraph, for any degree of a full non-recursive function.) Observe finally that there exist functions (for example the function $g_{0}$ of the following paragraph) which are not of the same degree as any full function on Davis' definition, though they are on ours. 
$\lambda x \Phi\left(g_{0}, x\right)$ is a restriction of the partial recursive function $\lambda x \Phi(n, x)$ for every completely computable $\Phi$ : but $c_{\gamma}$, being full and nonrecursive, cannot be a restriction of a partial recursive function. Hence $c$ is not partial recursive in $g_{0}$ in the sense of Davis.

We regard $f$ as effectively computable from $g$ in the intuitive sense if there exists a mechanical method by means of which every correct and no incorrect value of $f$ can be computed using only finitely many values of $g$. If we assume that this method can be formalized in some formal system with recursive rules of inference, we are led to the following amendment of Davis' definition.

$f$ is called partial recursive in $g$ if there is a recursively enumerable relation $R(x, y, t)$ for which

$$
f(x)=t \leftrightarrow(\exists y)\left(y^{[1]} \subseteq g \text { and } R(x, y, t)\right) .
$$

Trivially, if $f$ is partial recursive in $g$ in Davis' sense, it is in ours too. For $g$ full the converse holds (cf. the Corollary of Theorem $\mathrm{XIX}$ in [2, p. 331]) but by the immediately preceding counterexample not for $g$ arbitrary. Two functions are called strongly (Turing) equivalent if each is partial recursive in the other sense of Davis [1, p. 171]; weakly equivalent if each is partial recursive in the other in the sense of our definition. If two functions are strongly equivalent they are weakly equivalent; but not conversely by footnote 4 above. The equivalence classes relative to strong (weak) equivalence are called strong (weak) degrees. Not every strong degree contains a full function (footnote 4). Our main result in this note is that the same is true of weak degrees-in fact (cf. footnote 4) that "almost no" (weak) degree contains a full function. This will be established if we can prove the following theorem.

For each set $\alpha$, let $c_{\alpha}^{0}$ (the semicharacteristic function of $\alpha$ ) be that function which is 0 on $\alpha$ and undefined elsewhere. The class of all sets $\alpha$ for. which some full nonrecursive function is partial recursive in $c_{\alpha}^{0}$ in the sense of our definition is of first category. ${ }^{5} A$ fortiori the same is true of the class of all $\alpha$ for which some full nonrecursive function is partial recursive in $c_{\alpha}^{0}$ in Davis' sense, and of the class of all $\alpha$ for which $c_{\alpha}^{0}$ is strongly (weakly) equivalent to some full function.

For the proof, call $f$ partial recursive in $g$ with Gödel-number $i$ if (1) holds where $R$ is the $i$ th recursively enumerable relation in some canonical enumeration. If this is so we write $f=\left[\Phi_{i} g\right] .\left(\left[\Phi_{i} g\right]\right.$

${ }^{5}$ We use the topology standard in recursion theory, i.e. we identify sets (or their characteristic functions) with points of $\{0,1\} \aleph_{0}$, where $\{0,1\}$ is given the discrete topology. The collection of all classes $\{\alpha \mid \beta \subseteq \alpha$ and $\alpha \cap \gamma=\varnothing\}$, where $\beta$ and $\gamma$ are disjoint finite sets, forms a convenient basis of open classes. 
does not exist for all $i, g$ : in fact it exists if and only if

$$
y_{1}^{[1]}, y_{2}^{[1]} \subseteq g, R_{i}\left(x, y_{1}, t_{1}\right), R_{i}\left(x, y_{2}, t_{2}\right) \rightarrow t_{1}=t_{2}
$$

where $R_{i}$ is the $i$ th recursively enumerable relation.)

The theorem will follow if we can show that the class of all $\alpha$ for which $\left[\Phi_{i} c_{\alpha}^{0}\right]$ is full but not recursive is nowhere dense. Let then $N$ be any basic open class; we seek a subneighborhood $N_{0}$ of $N$ such that

$$
\alpha \in N_{0},\left[\Phi_{i} c_{\alpha}^{0}\right] \text { defined and full } \rightarrow\left[\Phi_{i} c_{\alpha}^{0}\right] \text { recursive. }
$$

Let $N=\{\alpha \mid \beta \subseteq \alpha$ and $\gamma \cap \alpha=\varnothing\}$. Then $N_{0}$ satisfying (2) is defined by cases as follows.

CASE I. $\left[\Phi_{i} c_{\gamma}^{0}\right]$ is full. Then set $N_{0}=N$. For if $\alpha$ satisfies the hypothesis of (2) we have $\alpha \subseteq \gamma^{\prime}, c_{\alpha}^{0} \subseteq c_{\gamma^{\prime}}^{0},\left[\Phi_{i} c_{\alpha}^{0}\right] \subseteq\left[\Phi_{i} c_{\gamma^{\prime}}^{0}\right]$. But since $\left[\Phi_{i} c_{\alpha}^{0}\right]$ is full, $\left[\Phi_{i} c_{\alpha}^{0}\right]=\left[\Phi_{i} c_{\gamma^{\prime}}^{0}\right]$ and is therefore recursive.

CASE II. $\left[\Phi_{i} c_{\gamma^{\prime}}^{0}\right]$ is defined, but not full. Again set $N_{0}=N$. For as in Case I, $\alpha \in N_{0} \rightarrow\left[\Phi_{i} c_{\alpha}^{0}\right] \subseteq\left[\Phi_{i} c_{\gamma^{\prime}}^{0}\right]$. But then $\left[\Phi_{i} c_{\alpha}^{0}\right]$ is not full either, and (2) is vacuously true.

CASE III. $\left[\Phi_{i} c_{\gamma}^{0}\right]$ undefined. This can only happen if

$$
\left(\exists y_{1} y_{2} t_{1} t_{2} x\right)\left(y_{1}^{[1]}, y_{2}^{[1]} \subseteq c_{\gamma}^{0}, \quad R_{i}\left(x, y_{1}, t_{1}\right), \quad R_{i}\left(x, y_{2}, t_{2}\right), \quad t_{1} \neq t_{2}\right) .
$$

Let $\delta$ be the union of the domains of $y_{1}^{[1]}$ and $y_{2}^{[1]}$ (so that $\delta \subseteq \gamma^{\prime}$ ). Then we can set $N_{0}=\{\alpha \mid \beta \cup \delta \subseteq \alpha$ and $\gamma \cap \alpha=\varnothing\}$. For $\alpha \in N_{0} \rightarrow \delta \subseteq \alpha \rightarrow y_{1}^{[1]}$, $y_{2}^{[1]} \subseteq c_{\alpha}^{0} \rightarrow\left[\Phi_{i} c_{\alpha}^{0}\right]$ undefined; and again (2) holds vacuously.

\section{BIBLIOGRAPHY}

1. M. Davis, Computability and unsolvability, New York, McGraw-Hill Book Company, Inc., 1958.

2. S. C. Kleene, Introduction to metamathematics, Princeton, D. Van Nostrand Company, Inc., 1952.

University of CALIFornia, Berkeley 